\title{
3-D imaging and illustration of the perfusive mouse islet sympathetic innervation and its remodelling in injury
}

\author{
Y.-C. Chiu • T.-E. Hua • Y.-Y. Fu • \\ P. J. Pasricha $\cdot$ S.-C. Tang
}

Received: 9 May 2012 / Accepted: 26 July 2012 /Published online: 30 August 2012

(C) Springer-Verlag 2012

\begin{abstract}
Aims/hypothesis Sympathetic nerves influence islet hormone levels in the circulation. Insights into islet sympathetic innervation and its remodelling in diabetes may impact future therapeutics. However, standard immunohistochemistry and microtome-based microscopy cannot provide an integral view of the islet neurovascular complex. We prepared transparent islet specimens to investigate the spatial relationship between sympathetic nerves, blood vessels and islet cells in normal, streptozotocin-injected and non-obese diabetic mouse models.

Methods Cardiac perfusion of fluorescent lectin was used to label pancreatic blood vessels. Tyrosine hydroxylase and nuclear staining were used to reveal islet sympathetic innervation and microstructure. Optical clearing (i.e. use of immersion solution to reduce scattering) was applied to enable
\end{abstract}

P.J. Pasricha and S.-C. Tang contributed equally to this work.

Electronic supplementary material The online version of this article (doi:10.1007/s00125-012-2699-6) contains peer-reviewed but unedited supplementary material, which is available to authorised users.

Y.-C. Chiu $\cdot$ T.-E. Hua $\cdot$ S.-C. Tang

Connectomics Research Center, National Tsing Hua University,

Hsinchu, Taiwan

Y.-C. Chiu $\cdot$ S.-C. Tang

Department of Chemical Engineering,

National Tsing Hua University,

Hsinchu, Taiwan

T.-E. Hua $\cdot$ S.-C. Tang $(\bowtie)$

Institute of Biotechnology, National Tsing Hua University,

101, Sec. 2, Kuang Fu Rd,

Hsinchu, Taiwan 30013

e-mail: sctang@life.nthu.edu.tw

Y.-Y. Fu • P. J. Pasricha

Division of Gastroenterology and Hepatology,

Johns Hopkins University School of Medicine,

Baltimore, MD, USA 3-dimensional confocal microscopy of islets to visualise the sympathetic neurovascular complex in space.

Results Unlike previously reported morphology, we observed perfusive intra-islet, perivascular sympathetic innervation, in addition to peri-islet contacts of sympathetic nerves with alpha cells and sympathetic fibres encircling the adjacent arterioles. The intra-islet axons became markedly prominent in streptozotocin-injected mice (2 weeks after injection). In non-obese diabetic mice, lymphocytic infiltration remodelled the peri-islet sympathetic axons in early insulitis.

Conclusions/interpretation We have established an imaging approach to reveal the spatial features of mouse islet sympathetic innervation. The neurovascular complex and sympathetic nerve-alpha cell contact suggest that sympathetic nerves modulate islet hormone secretion through blood vessels, in addition to acting directly on alpha cells. In islet injuries, sympathetic nerves undergo different remodelling in response to different pathophysiological cues.

Keywords Insulitis · Islet · Neurovascular complex · NOD mouse $\cdot$ Optical clearing $\cdot$ Streptozotocin $\cdot$ Sympathetic nerves $\cdot$ Vessel painting

$\begin{array}{ll}\text { Abbreviations } \\ \text { 2-D } & \text { 2-dimensional } \\ \text { 3-D } & \text { 3-dimensional } \\ \text { GFP } & \text { Green fluorescent protein } \\ \text { NOD } & \text { Non-obese diabetic } \\ \text { STZ } & \text { Streptozotocin } \\ \text { TH } & \text { Tyrosine hydroxylase }\end{array}$

\section{Introduction}

The autonomic nervous system influences pancreatic islet hormone secretion [1]. Parasympathetic nerves, for instance, 
stimulate insulin secretion, whereas activation of sympathetic nerves inhibits insulin and stimulates glucagon secretion to elevate glucose levels in the circulation [2-4]. Histological studies reveal that islets are richly innervated by autonomic nerves, which enter the islets with the vasculature [5-8]. Despite these observations, to date, investigators still lack a detailed neurovascular anatomy of islets to understand the control mechanism, even in animals [9, 10]. The difficulty is due to the intricate islet vascular and neural plexuses, which cannot be easily portrayed by the standard microtome-based 2-dimensional (2-D) microscopy. The condensed islet vasculature and dispersed islet innervation in space require substantial resolving power and sensitivity to capture and distinguish image signals.

To overcome the imaging hurdle, we previously developed a penetrative imaging method, based on preparation of transparent tissues (or 'optical clearing') [11, 12], for 3dimensional (3-D) imaging of the mouse pancreatic and intestinal microstructure and vasculature [13-15] and the human enteric nervous system [16, 17]. Photon penetration in the tissue was improved by the process of optical clearing (i.e. use of the immersion solution to reduce scattering as light travels in the specimen [18-20]) to allow for deeptissue microscopy. Here, in islet imaging, we employed the same penetrative 3-D microscopy with vessel painting (perfusion of blood vessels with fluorescent probes) [13, 21] and islet histology [7, 8, 22, 23] to provide a global and integral view of the islet sympathetic neurovascular complex.

Using the optically cleared tissues, for the first time we were able to see the sympathetic neurovascular complex at the centre of the mouse islet. The in-depth projection of the scanned volume reveals the perfusive intra-islet, perivascular sympathetic innervation in addition to the peri-islet axons and fibres encircling the adjacent arterioles. Furthermore, our optical method also revealed remodelling of the sympathetic innervation due to streptozotocin (STZ)-induced microcirculatory injury and lymphocytic infiltration in the non-obese diabetic (NOD) mouse model in the early phase of insulitis [24-27]. In this report, the development of our islet imaging approach and the functional and pathophysiological implications of the neurovascular network in health and disease are presented and discussed.

\section{Research design and methods}

Animals and tissue labelling Pancreases harvested from nestin-green fluorescent protein (GFP) transgenic mice $[13,28]$ and BALB/c mice (female; BioLASCO, Taipei, Taiwan) were used to acquire images of normal islet innervation. Pancreases harvested from STZ (Sigma, St Louis, MO, USA)-injected BALB/c mice (female, age 7 weeks; single i.p. STZ injection, $180 \mu \mathrm{g} / \mathrm{g}$ body weight; killed at 2 and 4 weeks after injection) and NOD mice (female, age $7-$ 8 and 10-12 weeks; National Laboratory Animal Center, Taipei, Taiwan) were used to acquire images of islet remodelling in early insulitis.

Glucose levels of STZ-injected BALB/c mice were measured on day 3 and weekly, and compared with those of normal and NOD mice. Hyperglycaemia caused by STZinduced islet injury was confirmed by recording a $6 \mathrm{~h}$ fasting glucose concentration above $150 \mathrm{mg} / \mathrm{dl}$ on day 3 and in later blood samples. Overall, four nestin-GFP transgenic mice, eight normal BALB/c mice, eight STZ-injected BALB/c mice and eight NOD mice were used to generate representative images. Animal care was consistent with the Guidelines for Animal Experiments, National Tsing Hua University, Taiwan.

Vessel painting $[13,21]$ was performed by cardiac perfusion of the lectin (wheat germ agglutinin)-Alexa Fluor 488 , 555 or 633 conjugate $(30 \mu \mathrm{g} / \mathrm{g}$ of body weight; Invitrogen, Carlsbad, CA, USA) followed by $4 \%$ paraformaldehyde perfusion fixation. Afterwards, pancreases were harvested and the vibratome sections of the tissue $(\sim 400 \mu \mathrm{m})$ were post-fixed in 4\% paraformaldehyde solution for $1 \mathrm{~h}$ at $25^{\circ} \mathrm{C}$. The fixed tissues were then immersed in $2 \%$ Triton-X 100 solution for $2 \mathrm{~h}$ at $25^{\circ} \mathrm{C}$ for permeabilisation.

Four different primary antibodies were used to immunolabel the tissues following the protocol outlined below. The antibodies used were: polyclonal rabbit anti-tyrosine hydroxylase (TH) (Millipore, Billerica, MA, USA), polyclonal chicken anti-TH (Abcam, Cambridge, MA, USA), monoclonal rabbit anti-glucagon (Epitomics, Burlingame, CA, USA) and polyclonal guinea pig anti-insulin (Gene Tex, Irvine, CA, USA) antibodies. Before applying the antibody, the tissue was rinsed in PBS. This was followed by a blocking step, incubating the tissue with a blocking buffer $(2 \%$ Triton X-100, 10\% normal goat serum and $0.02 \%$ sodium azide in PBS). The primary antibody was then diluted in the dilution buffer $(1: 50,0.25 \%$ Triton X-100, 1\% normal goat serum and $0.02 \%$ sodium azide in $\mathrm{PBS}$ ) to replace the blocking buffer and incubated for 1 day at $15^{\circ} \mathrm{C}$.

Alexa Fluor 647 conjugated goat anti-rabbit, Alexa Fluor 546 conjugated goat anti-chicken or Alexa Fluor 546 conjugated goat anti-guinea pig secondary antibody (1:200; Invitrogen) was used individually or in combination to reveal the immunostained structure(s). Afterwards, nuclear staining by propidium iodide $(50 \mu \mathrm{g} / \mathrm{ml}$; Invitrogen $)$ or SYTO 16 (5 $\mu \mathrm{M}$; Invitrogen) was performed at room temperature for $1 \mathrm{~h}$. The labelled specimens were then immersed in the optical-clearing solution FocusClear (CelExplorer, Hsinchu, Taiwan) overnight before being imaged via confocal microscopy [18].

Confocal microscopy The Zeiss LSM 510 Meta confocal microscope equipped with objective of $10 \times$ (optical section $10 \mu \mathrm{m}$; Z-axis increment $5 \mu \mathrm{m}$ ) and $20 \times$ (optical section 
$5 \mu \mathrm{m}$; Z-axis increment $2.5 \mu \mathrm{m}$ ) 'Fluar' lenses was used to acquire gross images of the mouse islet(s) in the pancreas. The $40 \times$ LD 'C-Apochromat' water immersion lenses (working distance $620 \mu \mathrm{m}$ ) were used to acquire highresolution images (optical section $3 \mu \mathrm{m}$; Z-axis increment $1.5 \mu \mathrm{m})$. Each confocal micrograph consisted of 1,024 (X) $\times$ $1,024(\mathrm{Y})$ pixels. The laser-scanning process was operated under the multi-track scanning mode to sequentially acquire signals in multiple channels, including the transmitted light channel. The Alexa Fluor 647-labelled structures were excited at $633 \mathrm{~nm}$ and the fluorescence was collected by the 650-710 nm band-pass filter. The lectin-Alexa Fluor 555labelled vessels and propidium iodide-labelled nuclei were excited at $543 \mathrm{~nm}$ and the signals were collected by the 560 $615 \mathrm{~nm}$ band-pass filter. The lectin-Alexa Fluor 488, GFP and SYTO 16 signals were excited at $488 \mathrm{~nm}$ and the fluorescence was collected by the $500-550 \mathrm{~nm}$ band-pass filter.

Image projection and analysis The LSM 510 software (Carl Zeiss, Jena, Germany), Zen software (Carl Zeiss) and Avizo 6.2 image reconstruction software (VSG, Burlington, MA, USA), were used for projection and analysis of the confocal images. Figures 1c, 1d, 2a (right panel), 2b, 2c (right panel), $3 \mathrm{a}$ and $3 \mathrm{~b}$ (second and fourth panels), $4 \mathrm{~b}, 4 \mathrm{c}$ and $5 \mathrm{~b}-\mathrm{d}$, and ESM Figs 1a, 2, 3b, 3c, 4 and 5 were derived from the projection module of the LSM 510 software. ESM Figs 1e and $\mathrm{g}$ and ESM Video 2 were derived from the Avizo projection algorithms using the 'Voltex' module to create the 3-D images. The 3-D projection function of Avizo was used to present the orthogonal view of the $\mathrm{X} / \mathrm{Y}, \mathrm{Y} / \mathrm{Z}$ and $\mathrm{X} / \mathrm{Z}$ planes of the image stack shown in Fig. 2c (left panel). ESM Videos 1, 5, 7, 10 and 12 were recorded using the Avizo's 'Movie Maker' function with the increase in display time in association with the depth of the optical section (displayed using the 'Ortho Slice' function). Feature extraction and image segmentation were performed by the 'Label Field' function of Avizo to collect the voxels of the islet and its sympathetic nerves and blood vessels to perform the density analysis shown in Table 1 . The $360^{\circ}$ presentation of the image stacks in ESM Videos 3, 4, 6, 8, 9 and 11 was derived from the 'Panorama' function of the LSM 510 software. In ESM Fig. 1d, the 'Profile Analysis' module of the Zen software was used to reveal the change in signal intensity along the pixel line at the centre of the micrograph [16].

\section{Results}

Vessel painting is compatible with neurohistology in deeptissue imaging with optical clearing To visualise the islet vasculature with high definition, we performed mouse cardiac perfusion of fluorescent lectin (vessel painting) to label the endothelial lining. We first examined the pancreases from nestin-GFP transgenic mice [13, 28], which carry high GFP levels in the exocrine acini, to help define the pancreatic exocrine-endocrine boundary. ESM Fig. 1a shows an in-depth projection of an islet-rich region in the pancreatic tail, in which the condensed islet capillaries can readily be seen. The deep-tissue imaging of islets was made possible by optical clearing (ESM Figs $1 b$ and c). As can be seen, the transparent tissue enables the islet and large blood vessels to be seen in both the transmitted light and fluorescence micrographs.

The signal-to-noise ratio and the 3-D projection of islet blood vessels are shown in ESM Figs 1d and e. The signal peaks, which specify the local maxima of the fluorescent signals, indicate the presence of blood vessels. Because islets have a concentrated vascular network, images with high signal-to-noise ratio are essential to identify and distinguish the microvessels in space (ESM Videos 1 and 2).

To image the neurovascular complex, ESM Figs $1 \mathrm{f}$ and $\mathrm{g}$ show that vessel painting is compatible with immunostaining of the sympathetic marker TH (a critical enzyme in the production of both dopamine and noradrenaline [norepinephrine]) to simultaneously visualise the blood vessels and the sympathetic nerve fibres. Specifically, we choose to present the high-density $\mathrm{TH}^{+}$sympathetic nerve fibres encircling an arteriole (potentially to regulate the blood flow). This example demonstrates that our imaging approach has the resolving power to distinguish adjacent nerve fibres with micrometer-level resolution, similar to what we have achieved on examining enteric nerves in the human ileum [16].

Perivascular sympathetic innervation in the mouse pancreatic islet To examine the islet sympathetic neurovascular complex, we first targeted the islet in the nestin-GFP transgenic mouse and applied vessel painting and TH staining to reveal the tissue network. Figure 1 is a gallery display of the staining result at the centre of a large islet with a diameter of approximately $350 \mu \mathrm{m}$. Three features are readily visible in the micrographs: (1) high GFP levels in the exocrine domain; (2) the fluorescently perfused islet vasculature; and (3) a subset of $\mathrm{TH}^{+}$endocrine cells residing next to the capillaries. When enlarged (Fig. 1a, enlargement), the gallery image also reveals the presence of $\mathrm{TH}^{+}$sympathetic axons and varicosities in close contact with the blood vessels.

To confirm the association, we stacked the 12 gallery images in Fig. 1a and traced the TH signals and their paths inside and around the islet (ESM Fig. 2a). As can be seen, the intra-islet TH signals connect in space, associate with blood vessels and extend from the exterior to the interior domain of the islet. ESM Fig. $2 b$ and ESM Videos 3 and 4 show the in-depth projections of the islet vasculature and the $\mathrm{TH}^{+}$sympathetic innervation. Three features of the 
Fig. 1 Intra-islet, perivascular $\mathrm{TH}^{+}$sympathetic innervation of the mouse islet. (a) Intra-islet sympathetic innervation and its association with vasculature. The panels show a gallery display of the vessel-painted islet vasculature (red) and $\mathrm{TH}^{+}$ sympathetic nerves (grey) in the nestin-GFP mouse (exocrine domain: green; increment: $2.5 \mu \mathrm{m}$ per section). The enlargement shows the perivascular presence of $\mathrm{TH}^{+}$axons and varicosities. Yellow arrows: intra-islet $\mathrm{TH}^{+}$signals. Cyan arrows: $\mathrm{TH}^{+}$signals around the adjacent arteriole. Yellow asterisks: examples of $\mathrm{TH}^{+}$ endocrine cells in contact with the capillaries. Similar neurovascular features can be seen in other gallery panels. (b) Close-up images of the intraislet, perivascular $\mathrm{TH}^{+}$sympathetic innervation. Mouse: wild-type BALB/c. The box in the second panel is enlarged to show capillary-associated varicosities and axon elongation (yellow arrows). (c) Projections of the islet vasculature, $\mathrm{TH}^{+}$innervation and nuclei. Yellow and cyan arrows indicate intra- and peri-islet axons, respectively. The yellow arrows in panels (b) and (c) indicate the same axons. Projection depth: $15 \mu \mathrm{m}$. (d) Projections of vasculature and $\mathrm{TH}^{+}$innervation in an islet hemisphere. Cyan arrows indicate adjacent arterioles encircled by $\mathrm{TH}^{+}$ fibres. Projection depth: $96 \mu \mathrm{m}$. A 360-degree projection of $\mathrm{TH}^{+}$innervation is shown in ESM Video 6

\section{a}

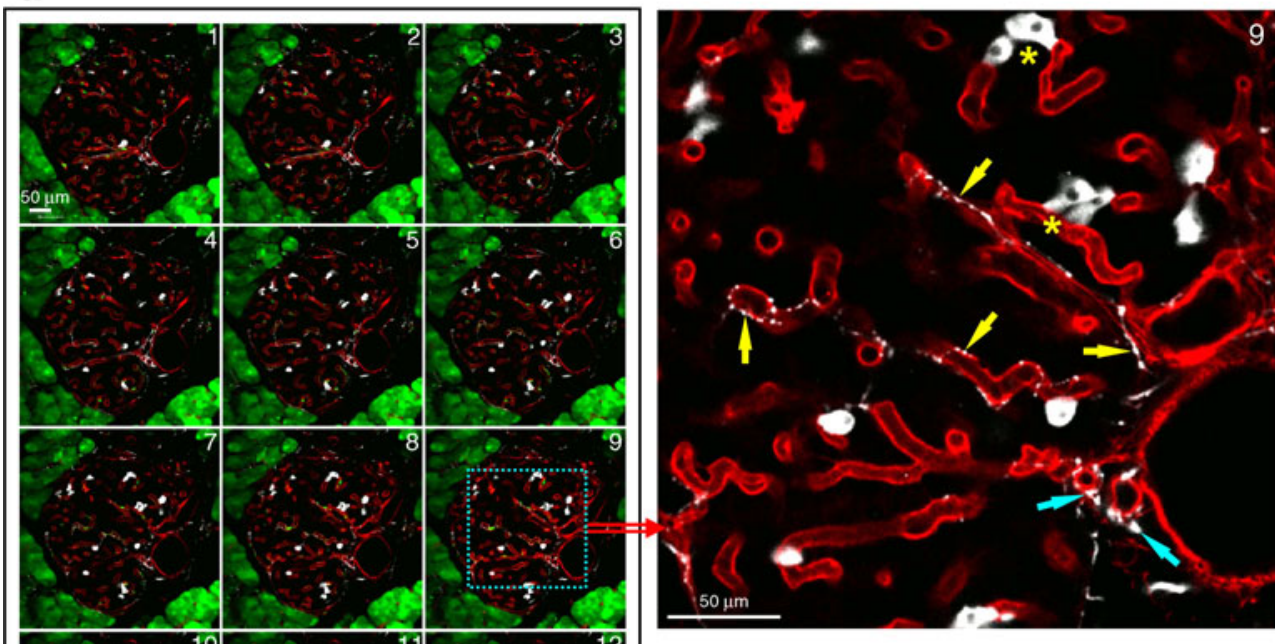

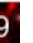$$
\text { . }
$$

b
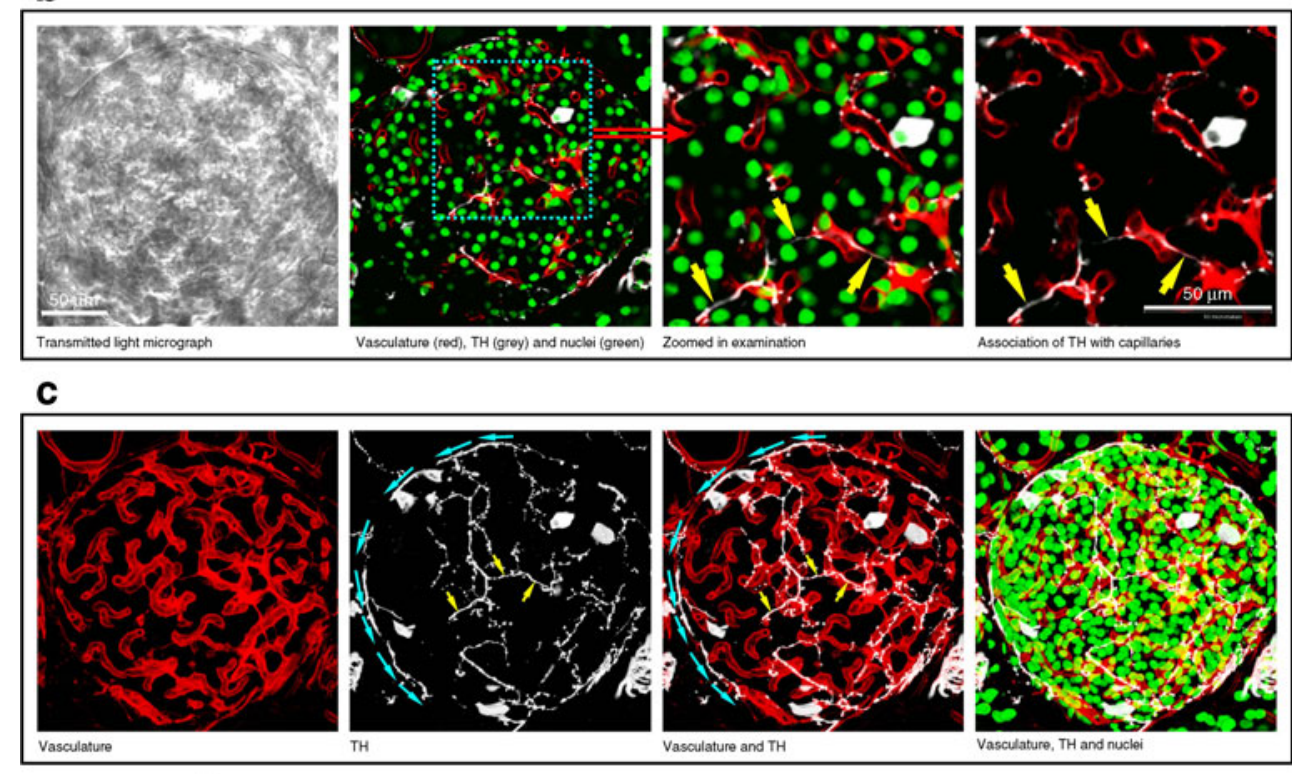

d

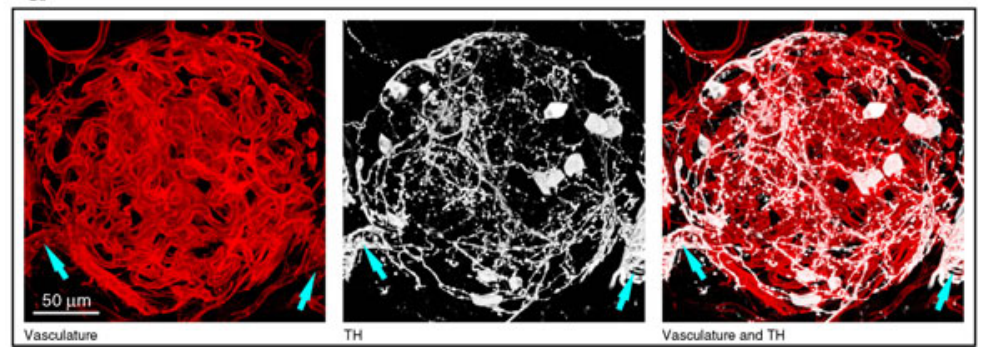

innervation are seen: (1) $\mathrm{TH}^{+}$fibres encircling the feeding arteriole, potentially to modulate islet blood flow; (2) $\mathrm{TH}^{+}$ axons reaching to the centre of the islet, in addition to their peri-islet presence; and (3) $\mathrm{TH}^{+}$varicosities in contact with the capillaries, suggesting a direct sympathetic input into the islet vascular system.

In the second example, we zoomed in to investigate a medium-size islet with a diameter of approximately $200 \mu \mathrm{m}$ 
a

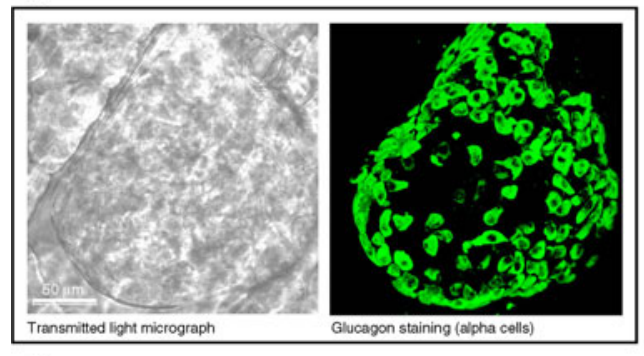

b

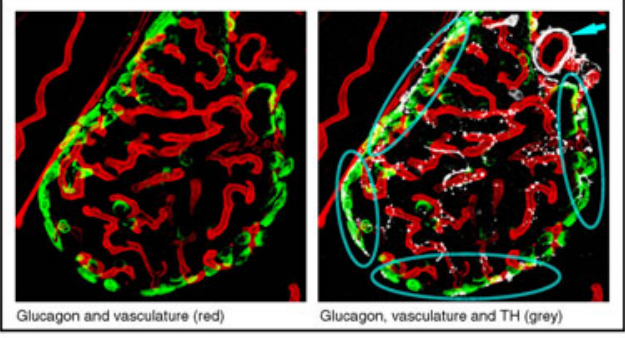

C

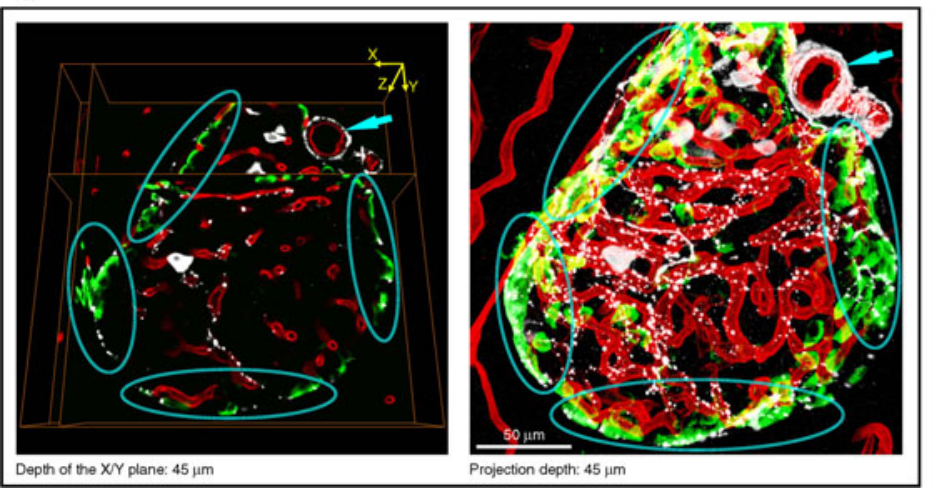

Fig. 2 Projections of glucagon, capillary and TH signals reveal three distinct patterns of mouse islet sympathetic innervation. (a) Transmitted light micrograph and projection of glucagon-expressing alpha cells at the islet periphery. Projection depth: $60 \mu \mathrm{m}$. (b) Projection of the islet middle section shows peri-islet TH signals in contact with alpha cells (squashed circles at the islet periphery). The arrow indicates the

in the BALB/c mouse. The transparent specimen allowed penetrative imaging of the capillary, TH and nuclear signals up to $96 \mu \mathrm{m}$ with high definition (ESM Video 5). Figure $1 \mathrm{~b}$ shows transmitted light and confocal micrographs of the islet, showing its spherical shape and the close association feeding arteriole. Projection depth: $15 \mu \mathrm{m}$. (c) Orthogonal and in-depth projections of glucagon, capillary and $\mathrm{TH}$ signals. The merged signals simultaneously reveal: (1) intra-islet, perivascular sympathetic innervation; (2) peri-islet contacts between $\mathrm{TH}^{+}$axons/varicosities and alpha cells (squashed circles); and (3) $\mathrm{TH}^{+}$fibres encircling the feeding arteriole (arrow). Projections were derived from the same image stack
Fig. 3 Comparison between normal and STZ-treated islets in morphologies of beta cells and $\mathrm{TH}^{+}$sympathetic innervation. (a) Insulin staining (blue) reveals that beta cells are abundant in the normal islet, yet the intra-islet TH signals (grey) are mostly associated with the capillaries (red) rather than found spread in the beta cell domain. (b) STZ injection induces beta cell destruction. Associated with this destruction, the chemically induced islet injury leads to an outgrowth of perivascular $\mathrm{TH}^{+}$nerves, particular in the islet core. Images of the two islets were taken and projected under the same magnification

\section{a}
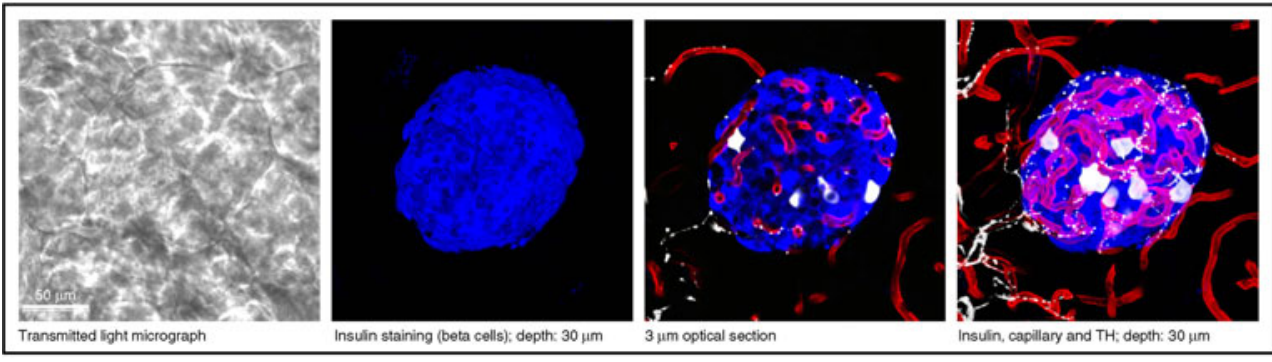

b
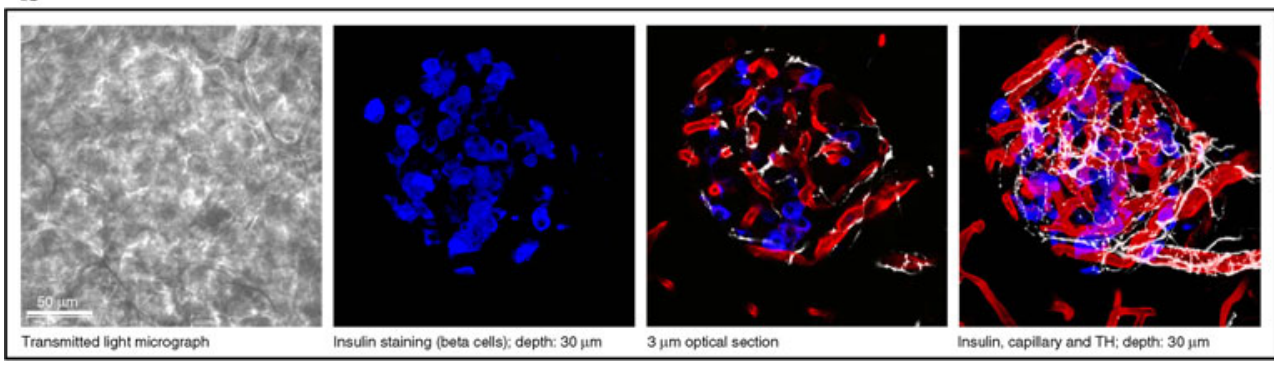
a

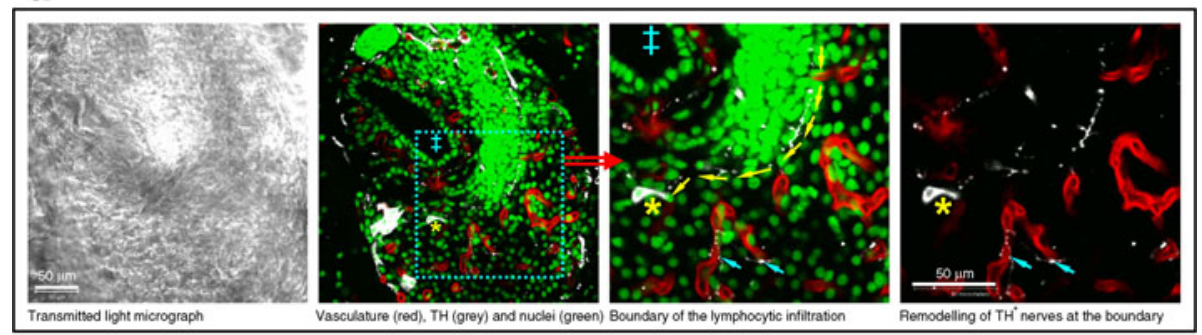

b

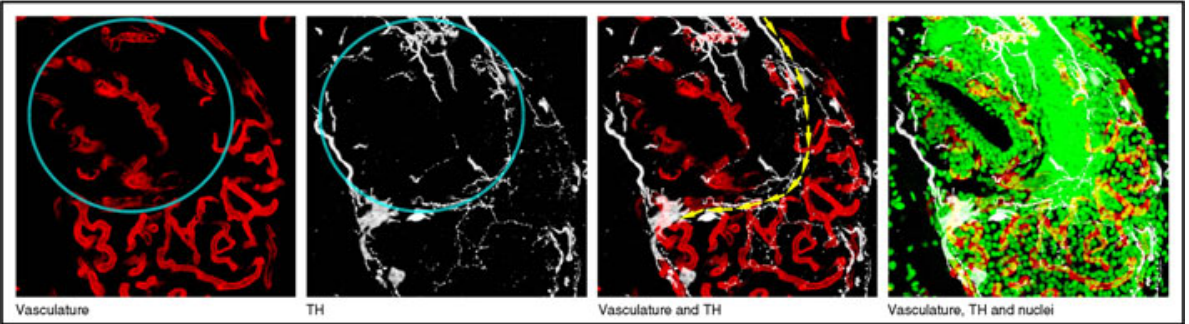

C

Fig. 4 Lymphocytic infiltration in NOD mice remodels the peri-islet sympathetic axons. (a) 2-D micrographs of islet lymphocytic infiltration. The box in the second panel is enlarged to show the boundary of the infiltrated area (yellow arrows). $\mathrm{TH}^{+}$nerves are rich at the front side of the boundary against the 'normal' domain. The double dagger indicates the lumen of a nearby pancreatic duct. The asterisk indicates a deformed $\mathrm{TH}^{+}$endocrine cell, potentially influenced by the infiltrated lymphocytes. Cyan arrows indicate perivascular $\mathrm{TH}^{+}$varicosities at the 'normal' domain. (b and c) Projections of the remodelled islet in the NOD mouse. Cyan circles in (b) indicate the 'inflammatory' domain with accumulation of lymphocytes. Vascular swelling is prominent in this domain. Yellow arrows indicate the front of the lymphocytic infiltration, which is associated with $\mathrm{TH}^{+}$nerves at the periphery of the 'normal' domain (yellow circles in (c)). Projection depths: 15 and $105 \mu \mathrm{m}$ in (b) and (c), respectively

islet 2 weeks after the STZ injection. We observed three abnormalities by comparing normal and diseased islets (comparison between Figs 1b, c and ESM Figs 3a, b): (1) higher nuclear density of the diseased islet; (2) a significant increase in the intra-islet axonal fibres around the capillaries relative to the normal association of varicosities with capillaries; and (3) a few $\mathrm{TH}^{+}$fibres being left without association with the blood vessels in the diseased islet. The abnormalities indicate ongoing remodelling of the islet microstructure, vasculature and innervation caused by STZ toxicity. The in-depth observation and panoramic projection of the increased $\mathrm{TH}^{+}$innervation are shown in ESM Fig. 3c and Videos 7 and 8.

Using paired immunostaining of insulin and TH, Fig. 3 compares the normal and STZ-treated islets in morphologies of beta cells and sympathetic innervation. In the normal islet, Fig. 3a shows that although the insulin-producing beta cells are abundant, a significant portion of them, particularly at the centre, do not contact with the $\mathrm{TH}^{+}$axons or
STZ injection stimulates islet sympathetic innervation Because the STZ injection causes islet microvascular injury, we sought to investigate whether the damage also influences capillary-associated sympathetic innervation. ESM Figs 3a and $b$ show 2-D micrographs and projections of the mouse 

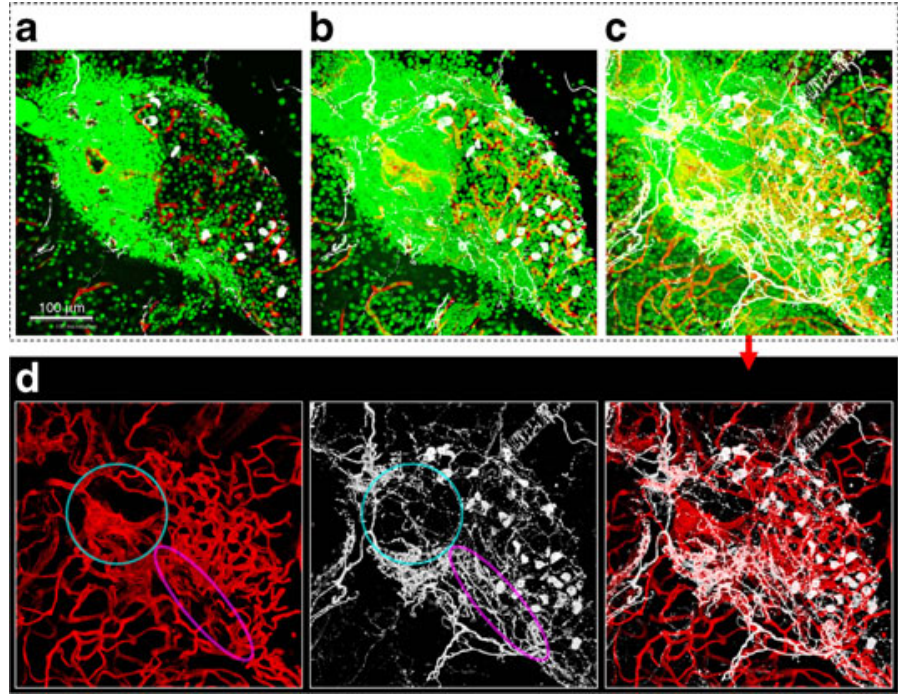

Fig. 5 Lymphocytic infiltration remodels the sympathetic neurovascular complex in the NOD mouse model (age 10 weeks). (a-c) 2-D micrograph and projections of islet lymphocytic infiltration. The progressive infiltration and accumulation of lymphocytes create a heterogeneous and remodelled $\mathrm{TH}^{+}$sympathetic neurovascular complex in the islet in comparison with those of normal (Fig. 1) and STZ-injected (Fig. 3) mice. Red: blood vessels. Grey: $\mathrm{TH}^{+}$nerves. Green: nuclei. (a)

varicosities, differing from the contact between alpha cells and $\mathrm{TH}^{+}$nerves at the islet periphery (Fig. 2).

In the STZ-treated islet, Fig. $3 \mathrm{~b}$ shows that the toxicity of STZ leads to beta cell destruction. This chemically induced injury has been shown to increase the neurotrophin content in the islet $[29,30]$, in which we observe an outgrowth of $\mathrm{TH}^{+}$nerves around the blood vessels, particularly in the islet core. Compared with the normal beta cells in the non-treated control (Fig. 3a), the remaining beta cells in the injured islet core appear to be under an increased sympathetic influence, which could in turn aggravate beta cell destruction under a stress response.
5- $\mu \mathrm{m}$ optical section; (b) $20-\mu \mathrm{m}$ projection; (c) $80-\mu \mathrm{m}$ projection. (d) Projections of the vasculature and $\mathrm{TH}^{+}$sympathetic nerves shown in (c). The 'inflammatory' domain (cyan circle) is associated with excessive accumulation of lymphocytes. The 'transition' area (magenta circle) has prominent $\mathrm{TH}^{+}$nerves against the 'normal' domain (the right side of the islet) in which the neurovascular morphology remains similar to that in the normal islet

Interestingly, because STZ injection increased the levels of $\mathrm{TH}^{+}$fibres to provide more immunostaining targets for detection, from a technical point of view this experiment also helped verify the presence of intra-islet sympathetic nerves, as presented in Fig. 1. ESM Fig. 4 and Video 9 present two additional examples of STZ-enhanced $\mathrm{TH}^{+}$ innervation in the islet. Images were taken 4 weeks after STZ injection.

Lymphocytic infiltration in NOD mice remodels the periislet sympathetic axons In the second islet remodelling experiment, we investigated whether islet lymphocytic

Table 1 Sympathetic nerve and blood vessel densities of islets of normal, STZ-injected and NOD mice

\begin{tabular}{lllll}
\hline Mouse model & Islets/animals $(n / n)$ & Corresponding images & Sympathetic nerve density & Blood vessel density $^{\mathrm{a}, \mathrm{b}}$ \\
\hline Normal (BALB/c) & $18 / 8$ & ESM Fig. 5a & $2.9 \pm 1.4$ & $20.1 \pm 6.4$ \\
STZ-injected (BALB/c) & $18 / 8$ & ESM Fig. 5b & $6.1 \pm 1.6^{\dagger}$ & $19.0 \pm 4.9^{\S}$ \\
2 weeks after injection & $12 / 6$ & & $6.3 \pm 1.7$ & $19.1 \pm 5.4$ \\
4 weeks after injection & $6 / 2$ & & $5.6 \pm 1.1$ & $18.7 \pm 4.2$ \\
NOD (in early insulitis) & $18 / 8$ & ESM Fig. 5c & $2.4 \pm 1.4^{\natural}$ & $15.3 \pm 7.3^{*}$ \\
\hline
\end{tabular}

Data are presented as mean $\pm \mathrm{SD}$

${ }^{\mathrm{a}} \mathrm{TH}^{+}$signals in endocrine cell bodies are excluded from the calculation of nerve density

${ }^{\mathrm{b}}\left(\mathrm{TH}^{+}\right.$voxels/voxels of islet $) \times 100 \%$

${ }^{\mathrm{c}}$ (Voxels of blood vessel/voxels of islet) $\times 100 \%$

${ }^{*} p<0.05,{ }^{\dagger} p<0.00001,{ }^{\S} p=0.54,{ }^{\natural} p=0.26$ versus normal control mice

No statistical difference between the two STZ groups (2 and 4 weeks after injection) 
infiltration in NOD mice remodels sympathetic innervation in the early phase of insulitis. Compared with the diffusive toxicity of STZ, lymphocytic infiltration first impacts the exterior part of the islet and then remodels or attacks the islet in a progressive fashion (Figs 4 and 5). Because of this heterogeneous nature, we observe two domains in or around the islet with distinct features (mice at age 7 and 10 weeks in Figs 4 and 5, respectively). In the 'inflammatory' domain, accumulation of lymphocytes is prominent and is associated with vascular swelling and destruction; the morphology is in sharp contrast to that of the 'normal' domain, in which the neurovascular complex appears intact and well-distributed in space.

Importantly, at the transition area of the two domains, the $\mathrm{TH}^{+}$nerves are prominent and can be found at the front of the infiltrated lymphocytes against the 'normal' domain (Figs 4a and b; ESM Videos 10-12 provide additional viewing depths and projection angles of the remodelled $\mathrm{TH}^{+}$innervation). The peri-'normal' domain axons and their intimate association with the infiltrated lymphocytes imply a previously overlooked participation of sympathetic nerves in early insulitis.

Quantitative analysis of the changes in islet sympathetic nerves and blood vessels in injury We have demonstrated remodelling of islet sympathetic innervation in injury. We next quantified the changes in islet sympathetic nerve and blood vessel densities by dividing their voxels with those of the islets in the acquired 3-D images. Table 1 lists the sympathetic nerve and blood vessel densities of islets of normal, STZ-injected and NOD mice. The projections of the 54 islets used in this analysis are categorised in ESM Fig. 5.

Compared with normal islets, islets of STZ-injected mice exhibit an outgrowth of sympathetic nerves with a 2.1-fold increase in nerve density. Note that this increase is not a transient response, as the nerve density remains elevated 4 weeks after injection. Regarding the islet vasculature, there is no statistical difference between normal and STZinjected mice in blood vessel density $(p=0.54)$.

In the NOD mice, lymphocytic infiltration in early insulitis decreases islet blood vessel density to $15.3 \%$ from $20.1 \%$ of the normal islet. However, peri-islet injury does not significantly change islet sympathetic nerve density $(p=0.26$, relative to that of the normal islet). This can be attributed to remodelling, rather than immediate destruction, of the periislet sympathetic nerves at the transition area between the infiltrated lymphocytes and the normal islet domain (the arrows in Fig. 4b and the magenta circle in Fig. 5d).

\section{Discussion}

The standard microtome-based 2-D tissue analysis cannot provide an integral and global perspective of islet vasculature and innervation. In this research, we combined vessel painting, neurohistology and deep-tissue microscopy with optical clearing to reveal the neurovascular complex of the islet. In particular, optical clearing generated transparent specimens, leading to penetrative imaging of the intricate neurovascular complex with high definition. This imaging approach simultaneously visualises islet microstructure, vasculature and innervation, and provides continuous and in-depth anatomic information for 3-D evaluation of the innervation pattern.

Using high-resolution images, we have demonstrated the presence of intra-islet, perivascular $\mathrm{TH}^{+}$sympathetic innervation in addition to peri-islet sympathetic axons and fibres encircling the arterioles adjacent to the islet. Our results differ from previous observations made by Rodriguez-Diaz et al of the mouse islet sympathetic innervation: we reveal intra-islet $\mathrm{TH}^{+}$axons and varicosities and their connection/ elongation in space and contacts with capillaries, which are absent in the mouse islet images presented previously [23]. Because Rodriguez-Diaz et al found sympathetic neurovascular contacts in human islets, they proceeded to emphasise species differences in innervation patterns. Our data, however, support species similarities in the innervation pattern: both mouse and human islets displayed a sympathetic neurovascular complex in the core, which is in line with the similar effect of autonomic activation on islet hormone release in animals and humans [3, 10, 31-34].

The potential reason for the discrepancy between the intra-islet $\mathrm{TH}^{+}$sympathetic innervation reported in this study and the lack of $\mathrm{TH}^{+}$signals inside mouse islets in the observations from Rodriguez-Diaz et al [23] might be due to the different optical properties of the islets used in the two studies. The transparent mouse islets that we prepared by optical clearing account for the detection of the $\mathrm{TH}^{+}$ neurovascular complex in the islet core, whereas the untreated islets used in the study of Rodriguez-Diaz et al study are prone to losing fluorescent signals because of scattering in the tissue. In neurohistology, light transmission is particularly important for detecting slender nerve fibres and small varicosities; substantial imaging sensitivity and resolving power are required to identify their presence and morphology in space. Because more tissue information can potentially be seen with transparent specimens, the importance of optical clearing in deep-tissue microscopy has recently been highlighted in neuroscience as well as in the imaging community. Work is proceeding on developing different clearing reagents and to establish different target tissues, including the gut and brain, to help understand the 3-D features of tissue architectures [16-18, 20].

The revealed neurovascular morphology in the current study supports the following three mechanisms, which may be used individually or in combination to influence islet hormone secretion in mice: (1) neurotransmitters are 
released and dispersed into the islet circulation through vascularly attached varicosities to regulate downstream endocrine cells in response to activation of sympathetic nerves (chemical stimulation/inhibition: indirect route); (2) while passing through the arteriole, the volume of islet blood flow is controlled by encircling sympathetic nerves to regulate the islet hormones flowing into the body circulation (mechanical modulation); and (3) because the peri-islet sympathetic axons are close to the mouse alpha cells (Figs 2b and c) [35], the sympathetic discharge could directly stimulate glucagon secretion, as suggested by Rodriguez-Diaz et al (chemical stimulation: direct route) [23].

In NOD mice, we have demonstrated remodelling of peri-islet sympathetic innervation in early insulitis, which is prior to the substantial loss of islet sympathetic nerves under diabetic conditions in the same animals reported by Taborsky et al [36]. Morphologically, our image data reveal heterogeneity of the islet microenvironment in early insulitis, which consists of a transition area with prominent sympathetic nerves at the interface between the inflammatory area and the normal islet domain. In the inflammatory area, the vascular swelling/damage shown in Figs 4 and 5 indicates an ongoing process of islet injury at the periphery associated with lymphocytic invasion, which would be expected to progress and lead to further tissue damage in severe insulitis. Note that at the beginning of the autoimmune attack, although the islet volume is eroded, the overall surface area of the islet actually increases-like an apple with a bite taken out - creating more transition areas at the boundary, which host the remodelled peri-islet sympathetic nerves (pathophysiologically, the increase in islet surface area creates additional lymphocyte-islet contacts for further lymphocytic infiltration). The phenomenon balances the loss of nerves in the damaged domain and is reflected in the quantitative analysis shown in Table 1, in which the islets of normal and NOD mice show no statistical difference in sympathetic nerve density.

The maintenance of islet sympathetic nerve density in early insulitis is also consistent with the functional data reported in the study by Taborsky et al of NOD mice [36]. In the tyramine stimulation of the sympathetic nerves, the non-diabetic group (age 18 weeks) maintained an active islet response to increase glucagon secretion $(260 \pm 32 \mathrm{ng} / \mathrm{l}$ increase in plasma concentration); the level was comparable to that of stimulated non-obese diabetes-resistant control mice $(170 \pm 24 \mathrm{ng} / \mathrm{l}$; age 13 weeks) and significantly higher than that of diabetic mice $(145 \pm 23 \mathrm{ng} / \mathrm{l}$; age 21 weeks). Seemingly, other studies also indicate that the glucagon response stays active early in STZ-induced diabetic animals [37, 38], despite their loss of beta cells - one major contributor to the glucagon response. Taken together, the morphologic and functional data suggest that the plasticity of sympathetic innervation is employed at the early phase of islet injury to compensate for partial loss of the islet function. However, this compensation cannot last long once the disease progresses to an advanced stage, with loss of sympathetic nerves becoming obvious under diabetic conditions.

In summary, we applied deep-tissue imaging with optical clearing to investigate islet sympathetic innervation in normal and diabetic mouse models. Prior to this research, tissue opacity and a lack of labelling tools have hindered observations of the islet neurovascular complex. In this research, we systematically built labelling and imaging methods to overcome the technical hurdles. Our observations of perfusive intra-islet, perivascular sympathetic innervation suggest that the islet vasculature plays a role in mediating the sympathetic discharge of islet hormone secretion. Future work will be aimed at extending the new tool for 3-D imaging of human islets, as well as mapping the parasympathetic nerves and other neural components, including the glia and sensory neurons, to examine islet innervation in health and diabetes in an integrated fashion.

Acknowledgements We thank Ms S-J Peng at National Tsing Hua University for excellent technical assistance, Dr A-S Chiang at National Tsing Hua University for technical support of confocal imaging and Dr G Enikolopov at Cold Spring Harbor Laboratory for supplying the nestinGFP transgenic mice.

Funding This work was supported by grants from the Taiwan National Health Research Institutes and National Science Council to S-CT.

Duality of interest The authors declare that there is no duality of interest associated with this manuscript.

Contribution statement Y-CC, T-EH and Y-YF contributed to the experimental design, participated in the analysis and interpretation of data, and revised the manuscript critically for intellectual content. PJP helped design the study and contributed to the writing in the medical and histological portions of the paper. S-CT directed the imaging project, helped design the study and contributed to the writing in the technical and histological portions of the paper. All authors approved the final version of the manuscript.

\section{References}

1. Woods SC, Porte D Jr (1974) Neural control of the endocrine pancreas. Physiol Rev 54:596-619

2. Ahren B, Wierup N, Sundler F (2006) Neuropeptides and the regulation of islet function. Diabetes 55:S98-S107

3. Ahren B (2000) Autonomic regulation of islet hormone secretion - implications for health and disease. Diabetologia 43:393-410

4. Brunicardi FC, Shavelle DM, Andersen DK (1995) Neural regulation of the endocrine pancreas. Int J Pancreatol 18:177-195

5. Coupland RE (1958) The innervation of pancreas of the rat, cat and rabbit as revealed by the cholinesterase technique. J Anat 92(Pt 1):143-149 
6. Miller RE (1981) Pancreatic neuroendocrinology: peripheral neural mechanisms in the regulation of the islets of Langerhans. Endocr Rev 2:471-494

7. Lindsay TH, Halvorson KG, Peters CM et al (2006) A quantitative analysis of the sensory and sympathetic innervation of the mouse pancreas. Neuroscience 137:1417-1426

8. Burris RE, Hebrok M (2007) Pancreatic innervation in mouse development and beta-cell regeneration. Neuroscience 150:592602

9. Gromada J, Franklin I, Wollheim CB (2007) Alpha-cells of the endocrine pancreas: 35 years of research but the enigma remains. Endocr Rev 28:84-116

10. Taborsky GJ Jr (2011) Islets have a lot of nerve! Or do they? Cell Metab 14:5-6

11. Fu YY, Tang SC (2010) At the movies: 3-dimensional technology and gastrointestinal histology. Gastroenterology 139:1100-1105

12. Smith K (2011) Neurogastroenterology: improving 3D imaging of the enteric nervous system. Nat Rev Gastroenterol Hepatol 8:600

13. Fu YY, Lu CH, Lin CW et al (2010) Three-dimensional optical method for integrated visualization of mouse islet microstructure and vascular network with subcellular-level resolution. J Biomed Opt 15:046018

14. Tang SC, Fu YY, Lo WF, Hua TE, Tuan HY (2010) Vascular labeling of luminescent gold nanorods enables 3-D microscopy of mouse intestinal capillaries. ACS Nano 4:6278-6284

15. Fu YY, Lin CW, Enikolopov G, Sibley E, Chiang AS, Tang SC (2009) Microtome-free 3-dimensional confocal imaging method for visualization of mouse intestine with subcellular-level resolution. Gastroenterology 137:453-465

16. Liu YA, Chen Y, Chiang AS, Peng SJ, Pasricha PJ, Tang SC (2011) Optical clearing improves the imaging depth and signal-to-noise ratio for digital analysis and three-dimensional projection of the human enteric nervous system. Neurogastroenterol Motil 23:e446e457

17. Liu YA, Chung YC, Pan ST et al (2012) 3-D illustration of network orientations of interstitial cells of Cajal subgroups in human colon as revealed by deep-tissue imaging with optical clearing. Am J Physiol Gastrointest Liver Physiol 302:G1099-G1110

18. Tuchin VV, Wang RK, Yeh AT (2008) Optical clearing of tissues and cells. J Biomed Opt 13:021101

19. Carl Zeiss Microimaging GmbH (2009) Visualizing the architecture of cells and tissues, Brochures for Laser Scanning Microscopy. Available from http://www.zeiss.de/micro, accessed 5 April 2012.

20. Hama H, Kurokawa H, Kawano H et al (2011) Scale: a chemical approach for fluorescence imaging and reconstruction of transparent mouse brain. Nat Neurosci 14:1481-1488

21. Ravnic DJ, Jiang X, Wolloscheck T et al (2005) Vessel painting of the microcirculation using fluorescent lipophilic tracers. Microvasc Res 70:90-96

22. Taylor-Fishwick DA, Bowman A, Korngiebel-Rosique M, Vinik AI (2008) Pancreatic islet immunoreactivity to the Reg protein INGAP. J Histochem Cytochem 56:183-191
23. Rodriguez-Diaz R, Abdulreda MH, Formoso AL et al (2011) Innervation patterns of autonomic axons in the human endocrine pancreas. Cell Metab 14:45-54

24. Beppu H, Maruta K, Kurner T, Kolb H (1987) Diabetogenic action of streptozotocin: essential role of membrane permeability. Acta Endocrinol (Copenh) 114:90-95

25. Papaccio G (1993) Insulitis and islet microvasculature in type 1 diabetes. Histol Histopathol 8:751-759

26. Enghofer M, Usadel KH, Beck O, Kusterer K (1997) Superoxide dismutase reduces islet microvascular injury induced by streptozotocin in the rat. Am J Physiol 273:E376-E382

27. Denis MC, Mahmood U, Benoist C, Mathis D, Weissleder R (2004) Imaging inflammation of the pancreatic islets in type 1 diabetes. Proc Natl Acad Sci USA 101:12634-12639

28. Mignone JL, Kukekov V, Chiang AS, Steindler D, Enikolopov G (2004) Neural stem and progenitor cells in nestin-GFP transgenic mice. J Comp Neurol 469:311-324

29. Larrieta ME, Vital P, Mendoza-Rodriguez A, Cerbon M, Hiriart M (2006) Nerve growth factor increases in pancreatic beta cells after streptozotocin-induced damage in rats. Exp Biol Med (Maywood) 231:396-402

30. Teitelman G, Guz Y, Ivkovic S, Ehrlich M (1998) Islet injury induces neurotrophin expression in pancreatic cells and reactive gliosis of peri-islet Schwann cells. J Neurobiol 34:304-318

31. Teff KL, Mattes RD, Engelman K (1991) Cephalic phase insulin release in normal weight males: verification and reliability. Am J Physiol 261:E430-E436

32. Havel PJ, Ahren B (1997) Activation of autonomic nerves and the adrenal medulla contributes to increased glucagon secretion during moderate insulin-induced hypoglycemia in women. Diabetes 46:801-807

33. Taborsky GJ Jr, Ahren B, Havel PJ (1998) Autonomic mediation of glucagon secretion during hypoglycemia: implications for impaired alpha-cell responses in type 1 diabetes. Diabetes 47:9951005

34. Gilliam LK, Palmer JP, Taborsky GJ Jr (2007) Tyramine-mediated activation of sympathetic nerves inhibits insulin secretion in humans. J Clin Endocrinol Metab 92:4035-4038

35. Cabrera O, Berman DM, Kenyon NS, Ricordi C, Berggren PO, Caicedo A (2006) The unique cytoarchitecture of human pancreatic islets has implications for islet cell function. Proc Natl Acad Sci USA 103:2334-2339

36. Taborsky GJ Jr, Mei Q, Hackney DJ, Figlewicz DP, LeBoeuf R, Mundinger TO (2009) Loss of islet sympathetic nerves and impairment of glucagon secretion in the NOD mouse: relationship to invasive insulitis. Diabetologia 52:2602-2611

37. Patel DG (1983) Lack of glucagon response to hypoglycemia in long-term experimental diabetic rats. Diabetes 32:55-60

38. Shi ZQ, Rastogi KS, Lekas M, Efendic S, Drucker DJ, Vranic M (1996) Glucagon response to hypoglycemia is improved by insulin-independent restoration of normoglycemia in diabetic rats. Endocrinology 137:3193-3199 